\title{
On the Functional Empirical Process and Its Application to the Mutual Influence of the Theil-Like Inequality Measure and the Growth
}

\author{
Pape Djiby Mergane ${ }^{1}$, Gane Samb Lo ${ }^{1,2}$ \\ ${ }^{1}$ LERSTAD, Université Gaston Berger de Saint-Louis, Saint-Louis, Sénégal \\ ${ }^{2}$ LSTA, Université Pierre et Marie Curie, Paris, France \\ Email: pdmergane@ufrsat.org, ganesamblo@yahoo.com
}

Received January 20, 2013; revised February 25, 2013; accepted March 5, 2013

Copyright (C) 2013 Pape Djiby Mergane, Gane Samb Lo. This is an open access article distributed under the Creative Commons Attribution License, which permits unrestricted use, distribution, and reproduction in any medium, provided the original work is properly cited.

\begin{abstract}
We set in this paper a coherent theory based on functional empirical processes that allows to consider both the poverty and the inequality indices in one Gaussian field in which the study of the influence of the one over the other is done. We use the General Poverty Index (GPI), that is a class of poverty indices gathering the most common ones and a functional class of inequality measures including the Entropy Measure, the Mean Logarithmic Deviation, the different inequality measures of Atkinson, Champernowne, Kolm and Theil called Theil-Like Inequality Measures (TLIM). Our results are given in a unified approach with respect to the two classes instead of their particular elements. We provide the asymptotic laws of the variations of each class over two given periods and the ratio of the variation and derive confidence intervals for them. Although the variances may seem somehow complicated, we provide $\mathrm{R}$ codes for their computations and apply the results for the pseudo-panel data for Senegal with a simple analysis.
\end{abstract}

Keywords: Functional Empirical Process; Asymptotic Normality; Welfare and Inequality Measure; Weak Laws; Pro and Anti-Poor Growth

\section{Introduction}

In many cases, one has to monitor a specific situation through some risk measure $J$ on some population. The variation of $J$ over time is called growth in case of negative variation and recession alternatively. This growth or recession is not itself sufficient to describe the improvement or deterioration of the situation. Often, the distribution of the underlying variable over the population should also be taken into account in order to check whether the growth concerns a great number of individuals or is rather concentrated on a few numbers of them.

In the particular case of welfare analysis, one may measure poverty (or richness) with the help of poverty indices $J$ based on the income variable $X$. Over two periods $s=1$ and $t=2$, we say that we have a gain against poverty when $\Delta J(s, t)=J(t)-J(s) \leq 0$, or simply a growth against poverty. Before claiming any victory, one must be sure that, meanwhile, the income did not become more unequally distributed, that is the appropriate ine- quality coefficient $I$ did not increase. One can achieve this by studying the ratio $R=\Delta J(s, t) / \Delta I(s, t)$.

To make the ideas more precise, let us suppose that we are monitoring the poverty scene on some population over the period time [1,2] and let $\left(X^{1}, X^{2}\right)$ be the income variable of that population at periods 1 and 2 . Let us consider one sample of $n \geq 1$ individuals or households, and observe the income couple $Z_{j}=\left(X_{j}^{1}, X_{j}^{2}\right)$, $j=1, \cdots, n$. For each period $i \in\{1,2\}$, we assume that $X^{i}$ is strictly positive, and we compute the poverty measure $J_{n}(i)$ and the inequality measure $I_{n}(i)$. We draw the attention of the reader that we consider here classes of measures both for poverty and inequality rather than specific ones. This leads to the very general results but requires extended notation.

For poverty, we consider the Generalized Poverty Index (GPI) introduced by Lo at al. [1] and Lo [2] as an attempt to gather a large class of poverty measures reviewed in Zheng [3] defined as follows for period $i$, 


$$
J_{n}(i)=\frac{A\left(Q_{n}(i), n, Z(i)\right)}{n B\left(Q_{n}(i)\right)} \sum_{j=1}^{Q_{n}(i)} w\left(\mu_{1} n+\mu_{2} Q_{n}(i)-\mu_{3} j+\mu_{4}\right) d\left(\frac{Z(i)-X_{j, n}^{i}}{Z(i)}\right)
$$

where $B\left(Q_{n}().\right)=\sum_{j=1}^{n} w(j), \quad Z($.$) is the income level$ representing the poverty line, $Q_{n}($.$) is the number of$ poor, $\mu_{1}, \mu_{2}, \mu_{3}$ and $\mu_{4}$ are constants, $A(u, v, s), w(t)$, and $d(y)$ are mesurable functions of

$(u, v, s) \in \mathbb{N} \times \mathbb{N} \times \mathbb{R}_{+}^{*} \quad t \in \mathbb{R}_{+}^{*}$, and $x \in(0,1)$. By particularizing the functions $A$ and $w$ and by giving fixed values to the $\mu_{i}^{\prime}$ s, we may find almost all the available indices, as we will do it later on. In the sequel, (1) will be called a poverty index (indices in the plural) or simply a poverty measure according to the economists' terminalogy.

This class includes the most popular indices such as those of Sen [4], Kakwani [5], Shorrocks [6], ClarkHemming-Ulph [7], Foster-Greer-Thorbecke [8], etc. See Lo [2] for a review of the GPI. From the works of many authors $\left([9,10]\right.$ for instance), $J_{n}(i)$ is an asymptotically sufficient estimate of the exact poverty measure

$$
J(i)=\int_{0}^{Z(i)} L\left(x, G_{i}\right) d\left(\frac{Z(i)-x}{Z(i)}\right) \mathrm{d} G_{i}(x)
$$

where $G_{i}$ is the distribution function of $X^{i}(i=1,2)$, and $L$ is some weight function.

As for the inequality measure, we use this Theil-like family, where we gathered the Generalized Entropy Measure, the Mean Logarithmic Deviation [11-13], the different inequality measures of Atkinson [14], Champernowne [15] and Kolm [16] in the following form:

$$
I_{n}(i)=\tau\left(\frac{1}{h_{1}\left(\mu_{n}(i)\right)} \frac{1}{n} \sum_{j=1}^{n} h\left(X_{j}^{i}\right)-h_{2}\left(\mu_{n}(i)\right)\right)
$$

where $\mu_{n}(i)=\frac{1}{n} \sum_{j=1}^{n} X_{j}^{i}$ denotes the empirical mean while $h, h_{1}, h_{2}$, and $\tau$ are measurable functions.

The inequality measures mentioned above are derived from (3) with the particular values of $\alpha, \tau, h, h_{1}$ and $h_{2}$ as described below for all $s>0$ :

\section{- Generalized Entropy}

$$
\begin{aligned}
& \alpha \neq 0, \alpha \neq 1, \tau(s)=\frac{s-1}{\alpha(\alpha-1)}, \\
& h(s)=h_{1}(s)=s^{\alpha}, h_{2}(s) \equiv 0 ;
\end{aligned}
$$

- Theil's measure:

$$
\tau(s)=s, h(s)=s \log (s), h_{1}(s)=s, h_{2}(s)=\log (s) ;
$$

- Mean Logarithmic Deviation

$$
\tau(s)=s, h(s)=h_{2}(s)=\log \left(s^{-1}\right), h_{1}(s) \equiv 1 ;
$$

- Atkinson's measure:

$$
\begin{aligned}
& \alpha<1 \text { and } \alpha \neq 0, \tau(s)=1-s^{1 / \alpha}, \\
& h(s)=h_{1}(s)=s^{\alpha}, h_{2}(s) \equiv 0 ;
\end{aligned}
$$

- Champernowne's measure:

$$
\tau(s)=1-\exp (s), h(s)=h_{2}(s)=\log (s), h_{1}(s) \equiv 1 ;
$$

- Kolm's measure:

$$
\begin{gathered}
\alpha>0, \tau(s)=\frac{1}{\alpha} \log (s), \\
h(s)=h_{1}(s)=\exp (-\alpha s), h_{2}(s) \equiv 0 .
\end{gathered}
$$

We will see below that $I_{n}(i)$ converges to the exact inequality measure

$$
I(i)=\tau\left(\frac{1}{h_{1}(\mu(i))} \int_{0}^{\infty} h(x) \mathrm{d} G_{i}(x)-h_{2}(\mu(i))\right)
$$

where $\mu(i)=\mathbb{E}\left(X^{i}\right)$ is the mathematical expectation of $X^{i}$ that we suppose to be finite here.

Each measure of the Theil-like family has its own particular properties, derived from the combination of different concepts. One may mention the concept of welfare criteria (Atkinson [14], Sen [17]), that of the analogy with analysis of risks (Harsanyi [18,19]; Rothschild and Stiglitz [20]), the complaints approach (Temkin [21]) etc. The Theil inequality itself finds all its interest in the information-theoretic idea following that of main components (Kullback [22]) and based on the three axioms (Zero-valuation of certainty, Diminishing-valuation of probability, Additivity of independent events). A deep review of such of individual properties for a number inequality measures can be found in Cowell $[13,23,24]$ for instance.

It is worth mentioning that the TLIM presented here is rather a mathematical form gathering of a number of different measures having different insights. Its main interest is to provide a general and uniform approach for dealing with both poverty and inequality measures in the same time and to avoid details and repetitions, in a coherent framework for useful comparison studies. In coming papers, the families presented by Cowell $[13,23,24]$ will be studied in similar ways.

The motivations stated above lead to the study of the behavior of

$$
\left(\Delta J_{n}(s, t), \Delta I_{n}(s, t)\right)
$$

as an estimate of the unknown value of

$$
(\Delta J(s, t), \Delta I(s, t)) \text {. }
$$

Precisely confidence intervals of 


$$
R(s, t)=\frac{\Delta J(s, t)}{\Delta I(s, t)}
$$

will be an appropriate set of tools for the study of the influence of each measure on the other.

To achieve our goal we need a coherent asymptotic theory allowing the handling of longitudinal data as it is the case here and a stochastic process approach leading to asymptotic subresults with the help of the continuity mapping theorem.

We find that the functional empirical process, in the modern setting of weak convergence theory, provides that coherent asymptotic theory.

Indeed, we use bidimensional functional empirical processes $\mathbb{G}_{n}$, and its stochastic Gaussian limit $\mathbb{G}$ to entirely describe the asymptotic behaviour of $\left(\Delta J_{n}(s, t), \Delta I_{n}(s, t)\right)$ in the Gaussian field of $\mathbb{G}$ and then find the law of $R_{n}(s, t)=\Delta J_{n}(s, t) / \Delta I_{n}(s, t)$ as our best achievement.

The remainder of the paper is organized as follows. In Section 2, we remind key definitions and properties for functional empirical processes, and we state the asymptotic representation of the GPI of Sall and Lo [25] stated in Theorem 1 that will be used later on. In Section 3, we give our main results and make some commentaries and data driven applications to Senegalese pseudo-panel data are considered while the proofs and the tables are postponed in an appendix Section 5. Section 4 concludes.

\section{Functional Empirical Process and Representation of GPI}

\subsection{A Brief Reminder on Functional Empirical Processes}

Let $Z_{1}, Z_{2}, \cdots, Z_{n}$ be a sequence of independent and identically distributed (i.i.d.) random elements defined on the probability space $(\Omega, \mathcal{A}, \mathbb{P})$, with values in some metric space $(S, d)$. Given a collection $\mathcal{F}$ of mesurable functions $f: S \rightarrow \mathbb{R}$, the functional empirical process $(F E P)$ is defined by:

$$
\forall f \in \mathcal{F}, \mathbb{G}_{n}(f)=\frac{1}{\sqrt{n}} \sum_{j=1}^{n}\left(f\left(Z_{j}\right)-\mathbb{E} f\left(Z_{j}\right)\right) .
$$

This process is widely studied in van der Vaart [26] for instance. It is directly seen that whenever $\mathbb{E}\left(f(Z)^{2}\right)<\infty$, one has

$$
\frac{1}{n} \sum_{j=1}^{n} f\left(Z_{j}\right) \rightarrow \mathbb{P}(f)=\mathbb{E}(f(Z)) \text { a.s. }
$$

and $\mathbb{G}_{n}(f) \rightarrow \mathcal{N}\left(0, \sigma_{f}^{2}\right)$ where

$$
\sigma_{f}^{2}=\mathbb{E}\left((f(Z)-\mathbb{P}(f))^{2}\right)<\infty,
$$

as consequences of the real Law of Large Numbers ( $L L N)$ and the real Central Limit Theorem (CLT).

When using the FEP, we may be interested in uniform $L L N$ 's and weak limits of the FEP considered as stochastic processes. This gives the so important results on Glivenko-Cantelli classes and Donsker ones. Let us define them here (for more details see [26,27]).

Since we may deal with non measurable sequences of random elements, we generally use the outer almost sure convergence defined as follows. Remind that a sequence $U_{n}$ converges outer almost surely to zero, denoted by $U_{n} \rightarrow 0$ a.s. ${ }^{\star}$, whenever there is a measurable sequence of measurable random variables $V_{n}$ such that

1) $\forall n,\left|U_{n}\right| \leq V_{n}$,

2) $V_{n} \rightarrow 0$ a.s.

The weak convergence generally holds in $\ell^{\infty}(\mathcal{F})$, the space of all bounded real functions defined on $\mathcal{F}$, equipped with the supremum norm

$$
\|x\|_{\mathcal{F}}=\sup _{f \in \mathcal{F}}|x(f)| .
$$

Definition 1 A class $\mathcal{F} \subset L_{1}(\mathbb{P})$ is a GlivenkoCantelli class for $\mathbb{P}$, if

$$
\begin{aligned}
& \lim _{n \rightarrow \infty} \mid \frac{1}{n} \sum_{j=1}^{n}\left(f\left(Z_{j}\right)-\mathbb{E} f\left(Z_{j}\right)\right) \|_{\mathcal{F}} \\
& =\limsup _{n \rightarrow \infty}\left|\frac{1}{n} \sum_{j=1}^{n}\left(f\left(Z_{j}\right)-\mathbb{E} f\left(Z_{j}\right)\right)\right|=0 \text { a.s. }{ }^{\star}
\end{aligned}
$$

Definition 2 A class $\mathcal{F} \subset L_{2}(\mathbb{P})$ is a Donsker class for $\mathbb{P}$, or $\mathbb{P}$-Donsker class if $\left\{\mathbb{G}_{n}(f) ; f \in \mathcal{F}\right\}$ converges in $\ell^{\infty}(\mathcal{F})$ to a centered Gaussian process $\{\mathbb{G}(f) ; f \in \mathcal{F}\}$ with covariance function

$$
\begin{aligned}
& \mathbb{E}(\mathbb{G}(f) \mathbb{G}(g)) \\
& =\int_{\mathbb{R}}(f(z)-\mathbb{E} f(z))(g(z)-\mathbb{E} g(z)) d \mathbb{P}(z) ; \\
& \forall f, g \in \mathcal{F} .
\end{aligned}
$$

Remark 1 When $S=\mathbb{R}$ and $\mathcal{F}=\left\{f_{t}=\mathbb{I}_{(-\infty, t]}, t \in \mathbb{R}\right\}$, $\mathbb{G}_{n}$ is the real empirical process and is denoted by $\alpha_{n}$.

In this paper, we only use finite-dimensional forms of the FEP, that is $\left(\mathbb{G}_{n}\left(f_{i}\right), i=1, \cdots, k\right)$. And then, any family $\left\{f_{i}, i=1, \cdots, k\right\}$ of measurable functions satisfying (5), is a Glivenko-Cantelli and a Donsker class, and hence

$$
\left(\mathbb{G}_{n}\left(f_{i}\right), i=1, \cdots, k\right) \stackrel{d}{\longrightarrow}\left(\mathbb{G}\left(f_{1}\right), \mathbb{G}\left(f_{2}\right), \cdots, \mathbb{G}\left(f_{k}\right)\right)
$$

where $\mathbb{G}$ is the Gaussian process, defined in Definition 2.

We will make use of the linearity property of both $\mathbb{G}_{n}$ and $\mathbb{G}$. Let $f_{1}, \cdots, f_{k}$ be measurable functions satisfying (5) and $a_{i} \in \mathbb{R}, i=1, \cdots, k$, then

$$
\sum_{j=1}^{k} a_{j} \mathbb{G}_{n}\left(f_{j}\right)=\mathbb{G}_{n}\left(\sum_{j=1}^{k} a_{j} f_{j}\right) \stackrel{d}{\longrightarrow} \mathbb{G}\left(\sum_{j=1}^{k} a_{j} f_{j}\right) .
$$


The materials defined here, when used in a smart way, lead to a simple handling the problem tackled here.

\subsection{Representation of the GPI}

In this paper, we use the GPI in a unified approach that leads to an asymptotic representation for a large class of indices classified in three kinds.

First we consider the threshold condition:

(H1) There exist $\beta>0$ and $0<\xi<1$ such that,

$$
0<\beta<G(Z)<\xi<1 \text {. }
$$

Next we have form conditions (on the indices):

(H2a) There exist a function $h(p, q)$ where $(p, q) \in \mathbb{N}^{2}$ and a function $c(s, t)$ where $(s, t) \in(0,1)^{2}$ such that, when $n \rightarrow+\infty$,

$$
\begin{aligned}
& \max _{1 \leq j \leq Q} \mid A(n, Q) h^{-1}(n, Q) w\left(\mu_{1} n+\mu_{2} Q-\mu_{3} j+\mu_{4}\right) \\
& -c(Q / n, j / n) \mid=o_{\mathbb{P}}\left(n^{-1 / 2}\right) ;
\end{aligned}
$$

(H2b) There exists a function $\pi(s, t)$ with $(s, t) \in \mathbb{R}^{2}$ such that, when $n \rightarrow+\infty$,

$$
\max _{1 \leq j \leq Q}\left|w(j) h^{-1}(n, Q)-\frac{1}{n} \pi(Q / n, j / n)\right|=o_{\mathbb{P}}\left(n^{-3 / 2}\right) .
$$

Further we need regularity conditions on $c$ and $\pi$ :

(H3) The functions $c(\cdot)$ and $\pi(\cdot)$ have uniformly continuous partial derivatives, that is

$$
\lim _{(k, l) \rightarrow(0,0)} \sup _{(x, y) \in(0,1)^{2}}\left|\frac{\partial c}{\partial y}(x+l, y+k)-\frac{\partial c}{\partial y}(x, y)\right|=0
$$

and

$$
\lim _{(k, l) \rightarrow(0,0)} \sup _{\beta \leq x \leq \xi, y \in(0,1)}\left|\frac{\partial c}{\partial x}(x+l, y+k)-\frac{\partial c}{\partial x}(x, y)\right|=0 ;
$$

(H4) The functions $y \rightarrow \frac{\partial c}{\partial y}(x, y)$ and $y \rightarrow \frac{\partial \pi}{\partial y}(x, y)$ are monotonous.

(H5) The distribution function $G$ is increasing.

(H6) There exist $H_{0}>0$ and $H_{\infty}<+\infty$ such that

$$
H_{0}<H_{c}(G)=\int_{0}^{+\infty} c(G(Z), G(y)) \gamma(y) \mathrm{d} G(y)<H_{\infty},
$$

and

$$
H_{0}<H_{\pi}(G)=\int_{0}^{+\infty} \pi(G(Z), G(y)) e(y) \mathrm{d} G(y)<H_{\infty}
$$

where $\quad \gamma(x)=d\left(\frac{Z-x}{Z}\right) \mathbb{I}_{(x \leq Z)}$ and $\quad e(x)=\mathbb{I}_{(x \leq Z)} \quad$ for $x \in \mathbb{R}$.

Based on these hypotheses, we put

$$
J(G)=H_{c}(G) / H_{\pi}(G),
$$

$$
\begin{aligned}
g(\cdot)= & H_{\pi}^{-1}(G) g_{c}(\cdot)-H_{c}(G) H_{\pi}^{-2}(G) g_{\pi}(\cdot) \\
& +K(G) e(\cdot),
\end{aligned}
$$

with

$$
\begin{gathered}
g_{c}(\cdot)=c(G(Z), G(\cdot)) \gamma(\cdot), g_{\pi}(\cdot)=\pi(G(Z), G(\cdot)) e(\cdot), \\
K(G)=H_{\pi}^{-1}(G) K_{c}(G)-H_{c}(G) H_{\pi}^{-2}(G) K_{\pi}(G)
\end{gathered}
$$

where

$$
\begin{gathered}
K_{c}(G)=\int_{0}^{1} \frac{\partial c}{\partial x}(G(Z), s) \gamma\left(G^{-1}(s)\right) \mathrm{d} s, \\
K_{\pi}(G)=\int_{0}^{1} \frac{\partial \pi}{\partial x}(G(Z), s) e\left(G^{-1}(s)\right) \mathrm{d} s, \\
v(\cdot)=H_{\pi}^{-1}(G) \nu_{c}(\cdot)-H_{c}(G) H_{\pi}^{-2}(G) v_{\pi}(\cdot),
\end{gathered}
$$

and

$$
\begin{aligned}
& v_{c}(\cdot)=\frac{\partial c}{\partial y}(G(Z), G(\cdot)) \gamma(\cdot), \\
& v_{\pi}(\cdot)=\frac{\partial \pi}{\partial y}(G(Z), G(\cdot)) e(\cdot) .
\end{aligned}
$$

Now recall the functional empirical process

$$
\alpha_{n}(g)=\frac{1}{\sqrt{n}} \sum_{j=1}^{n} g\left(X_{j}\right)-\mathbb{E} g\left(X_{j}\right)
$$

and introduce

$$
\beta_{n}(v)=\frac{1}{\sqrt{n}} \sum_{j=1}^{n}\left\{G_{n}\left(X_{j}\right)-G\left(X_{j}\right)\right\} v\left(X_{j}\right),
$$

the reduced process of Sall et Lo (see [25]).

The representation results of [25] for the GPI is the following.

Theorem 1 Suppose that (H1)-(H6) are true, then we have the following representation

$$
\sqrt{n}\left(J_{n}(G)-J(G)\right)=\alpha_{n}(g)+\beta_{n}(v)+o_{\mathbb{P}}(1) .
$$

Although these conditions may appear complicated, they are simple to check in usual cases with the popular poverty measures. We will see this in Section 3.

We are going to state our main results.

\section{Results and Commentaries}

\subsection{Notations}

Let us consider the following Renyi representations. Let $\left\{U_{j}\right\}_{j=1, \cdots, n}$ and $\left\{V_{j}\right\}_{j=1, \cdots, n}$ two sequences of independent uniform rv's on $D=(0,1)$. Then we have the representation, meant as equalities in distribution:

$$
X_{j}^{1}=G_{1}^{-1}\left(U_{j}\right) \text { and } X_{j}^{2}=G_{2}^{-1}\left(V_{j}\right), j \in\{1, \cdots, n\}
$$

where $G_{i}^{-1}$ is the generalized inverse of $G_{i}$. We sup- 
pose that $G_{i}$ is continuous. The copula associated with the couple $\left(X^{1}, X^{2}\right)$ is defined by

$$
C(u, v)=G_{1,2}\left(G_{1}^{-1}(u), G_{2}^{-1}(v)\right), \forall(u, v) \in D^{2},
$$

where $G_{1,2}$ is the joint distribution function of $\left(X^{1}, X^{2}\right)$.

Next we consider the bidimensional functional empirical process based on $\left\{\left(U_{j}, V_{j}\right)\right\}_{j=1, \ldots, n}$, for some Donsker class $\mathcal{F}$ :

$$
\forall f \in \mathcal{F}, \mathbb{G}_{n}(f)=\frac{1}{\sqrt{n}} \sum_{j=1}^{n}\left(f\left(U_{j}, V_{j}\right)-\mathbb{P}_{(U, V)}(f)\right) ;
$$

and the limiting centered Gaussian stochastic process $\mathbb{G}$, its variance-covariance function defined by, for $(f, g) \in \mathcal{F}^{2}$ :

$$
\begin{aligned}
& \mathbb{E}(\mathbb{G}(f) \mathbb{G}(g)) \\
& =\mathbb{P}_{(U, V)}\left(\left(f-\mathbb{P}_{(U, V)}(f)\right)\left(g-\mathbb{P}_{(U, V)}(g)\right)\right) \\
& =\int_{D^{2}}\left(f(u, v)-\mathbb{P}_{(U, V)}(f)\right)\left(g(u, v)-\mathbb{P}_{(U, V)}(g)\right) \mathrm{d} C(u, v)
\end{aligned}
$$

where

$$
\mathbb{P}_{(U, V)}(f)=\mathbb{E}(f(U, V))=\int_{D^{2}} f(u, v) \mathrm{d} C(u, v) .
$$

Now we introduce the following notations based on the functions $\tau, h, h_{1}, h_{2}$ of (3) and on the functions $g$ and $v$ of Theorem 1 . The subscript $i$ refers to the period. The following series of notations are about the variation of the inequality measures and are listed below. Let us first denpte:

$$
B_{n}(i)=\frac{1}{n} \sum_{j=1}^{n} h\left(X_{j}^{i}\right), B(i)=\int_{0}^{\infty} h(x) \mathrm{d} G_{i}(x) ;
$$

and next, for all $(u, v) \in D^{2}$,

$$
\tilde{f}_{i}(u, v)=G_{i}^{-1} \circ \Pi_{i}(u, v)
$$

where $\Pi_{i}$ is the $i^{\text {th }}$ projection on $(0,1)$,

$$
f_{i, h}(u, v)=h \circ \tilde{f}_{i}(u, v) \text {. }
$$

And finally,

$$
\begin{aligned}
F_{i, I}^{\star}(u, v)= & \frac{K_{i}}{h_{1}(\mu(i))} f_{i, h}(u, v) \\
& -K_{i}\left(\frac{B(i) h_{1}^{\prime}(\mu(i))}{h_{1}^{2}(\mu(i))}+h_{2}^{\prime}(\mu(i))\right) \tilde{f}_{i}(u, v)
\end{aligned}
$$

with $K_{i}=\tau^{\prime}\left(\frac{B(i)}{h_{1}(\mu(i))}-h_{2}(\mu(i))\right)$ and

$F_{I}^{\star}(u, v)=F_{2, I}^{\star}(u, v)-F_{1, I}^{\star}(u, v)$,

where $h_{1}^{\prime}, h_{2}^{\prime}$ and $\tau^{\prime}$ are respectively the derivatives of the functions $h_{1}, h_{2}$ and $\tau$.

For our results on the variation of the GPI, we need the functions $g_{i}$ and $v_{i}$ provided by the representation of
Theorem 1. Put accordingly with these functions:

$$
g_{i}(x)=c\left(G_{i}(x)\right) q_{i}(x) \text { and } v_{i}(s)=c^{\prime}(s) q_{i}\left(G_{i}^{-1}(s)\right) \text {. }
$$

We define for all $(u, v) \in D^{2}$

$$
f_{i, s}(u, v)=\Pi_{i}\left(\mathbb{I}_{(o, s)}(u), \mathbb{I}_{(o, s)}(v)\right)
$$

where $\mathbb{I}_{(o, s)}$ is the indicator function on the set $(0, s)$,

$$
F_{i, J}^{\star}(u, v)=g_{i} \circ \tilde{f}_{i}(u, v)=g_{i} \circ G_{i}^{-1} \circ \Pi_{i}(u, v),
$$

and

$$
F_{J}^{\star}(u, v)=F_{2, J}^{\star}(u, v)-F_{1, J}^{\star}(u, v) .
$$

\subsection{Main Theorems}

We are now able to state our theorems. The first concerns the variation of the inequality measure.

Theorem 2 Let $\mu(i)$ finite for $i=1,2$ and let each $h_{i}$ continuously differentiable at each $\mu(i), i=1,2$. Let $\mathbb{P}_{(U, V)}\left(F_{I}^{\star 2}\right)<\infty$, then we have the following convergence as $n \rightarrow \infty$

$$
\sqrt{n}\left(\Delta I_{n}(1,2)-\Delta I(1,2)\right) \rightarrow_{d} \mathcal{N}\left(0, \Gamma_{I}(1,2)\right)
$$

where $\rightarrow_{d}$ stands for the convergence in distribution and

$$
\Gamma_{I}(1,2)=\int_{D^{2}}\left(F_{I}^{\star}(u, v)-\mathbb{P}_{(U, V)}\left(F_{I}^{\star}\right)\right)^{2} \mathrm{~d} C(u, v) .
$$

The second concerns the variation of the GPI.

Theorem 3 Let $\mu(i)$ finite for $i=1,2$. Suppose that $\mathbb{P}_{(U, V)}\left(\left(f_{1, s}\right)^{2}\right), \quad \mathbb{P}_{(U, V)}\left(\left(f_{2, s}\right)^{2}\right)$ and $\mathbb{P}_{(U, V)}\left(F_{J}^{\star 2}\right)$ are finite. Then

$$
\begin{aligned}
& \sqrt{n}\left(\Delta J_{n}(1,2)-\Delta J(1,2)\right) \\
& \rightarrow_{d} \mathbb{G}\left(F_{J}^{\star}\right)+\int_{D}\left(\mathbb{G}\left(f_{2, s}\right) v_{2}(s)-\mathbb{G}\left(f_{1, s}\right) v_{1}(s)\right) \mathrm{d} s
\end{aligned}
$$

which is a centered Gaussian process of variance-covariance function:

$$
\Gamma_{J}(1,2)=\Gamma_{1}(1,2)+\Gamma_{2}(1,2)+2 \Gamma_{3}(1,2)
$$

where

$$
\begin{gathered}
\Gamma_{1}(1,2)=\int_{D^{2}}\left(F_{J}^{\star}(u, v)-\mathbb{P}_{(U, V)}\left(F_{J}^{\star}\right)\right)^{2} \mathrm{~d} C(u, v) ; \\
\Gamma_{2}(1,2)=\gamma_{1}-2 \gamma_{2}+\gamma_{3}
\end{gathered}
$$

with

$$
\begin{aligned}
& \gamma_{1}=\int_{D^{2}} v_{2}(s) v_{2}(t)(\min \{s, t\}-s t) \mathrm{d} s \mathrm{~d} t \\
& \gamma_{2}=\int_{D^{2}} v_{2}(s) v_{1}(t)(C(t, s)-s t) \mathrm{d} s \mathrm{~d} t \\
& \gamma_{3}=\int_{D^{2}} v_{1}(s) v_{1}(t)(\min \{s, t\}-s t) \mathrm{d} s \mathrm{~d} t
\end{aligned}
$$

and 


$$
\begin{aligned}
\Gamma_{3}(1,2)= & \int_{D}\left\{v_{2}(s) \int_{(0,1) \times(0, s)} F_{J}^{\star}(u, v) \mathrm{d} C(u, v)\right. \\
& \left.-v_{1}(s) \int_{(0, s) \times(0,1)} F_{J}^{\star}(u, v) \mathrm{d} C(u, v)\right\} \mathrm{d} s \\
& -\mathbb{P}_{(U, V)}\left(F_{J}^{\star}\right) \int_{D} s\left(v_{2}(s)-v_{1}(s)\right) \mathrm{d} s .
\end{aligned}
$$

Thus last one handles the ratio of the two variations.

Theorem 4 Supposing that the above mentioned hypotheses are true, then

$$
\begin{aligned}
& \left(\sqrt{n}\left(\Delta J_{n}(1,2)-\Delta J(1,2)\right), \sqrt{n}\left(\Delta I_{n}(1,2)-\Delta I(1,2)\right)\right)^{t} \\
& \rightarrow{ }_{d} \mathcal{N}_{2}(0, \Sigma),
\end{aligned}
$$

with

$$
\Sigma=\left(\begin{array}{cc}
\Gamma_{J}(1,2) & \Gamma_{I, J}(1,2) \\
\Gamma_{I, J}(1,2) & \Gamma_{I}(1,2)
\end{array}\right)
$$

and

$$
\begin{aligned}
\Gamma_{I, J}(1,2)= & \mathbb{P}_{(U, V)}\left(\left(F_{I}^{\star}-\mathbb{P}_{(U, V)}\left(F_{I}^{\star}\right)\right)\left(F_{J}^{\star}-\mathbb{P}_{(U, V)}\left(F_{J}^{\star}\right)\right)\right) \\
& +\int_{D}\left\{v_{2}(s) \int_{(0,1) \times(0, s)} F_{I}^{\star}(u, v) \mathrm{d} C(u, v)\right\} \mathrm{d} s \\
& -\int_{D}\left\{v_{1}(s) \int_{(0, s) \times(0,1)} F_{I}^{\star}(u, v) \mathrm{d} C(u, v)\right\} \mathrm{d} s \\
& +\mathbb{P}_{(U, V)}\left(F_{I}^{\star}\right) \int_{D} s\left(v_{1}(s)-v_{2}(s)\right) \mathrm{d} s .
\end{aligned}
$$

In this case, let

$$
R=\frac{\Delta J(1,2)}{\Delta I(1,2)}, a=\frac{1}{\Delta I(1,2)} \text { and } b=\frac{\Delta J(1,2)}{(\Delta I(1,2))^{2}},
$$

then we have $\sqrt{n}\left\{R_{n}(1,2)-R(1,2)\right\}$ tends to a functional Gaussian process

$$
\begin{aligned}
& a\left(\mathbb{G}\left(F_{J}^{\star}\right)+\int_{D}\left(v_{2}(s) \mathbb{G}\left(f_{2, s}\right)-v_{1}(s) \mathbb{G}\left(f_{1, s}\right)\right) \mathrm{d} s\right) \\
& -b \mathbb{G}\left(F_{I}^{\star}\right) ;
\end{aligned}
$$

of covariance function

$$
\Gamma(1,2)=a^{2} \Gamma_{J}(1,2)+b^{2} \Gamma_{I}(1,2)-2 a b \Gamma_{I, J}(1,2) .
$$

\subsection{Commentaries}

First of all, the results cover a large class of poverty measures and inequality indices. This explains why the notations seem heavy. Secondly, the variances of the limiting Gaussian processes seem also somehow tricky. But all of them are easily handled by modern computation means. We are going to particularise our results for famous measures and provide workable software codes for the computations.

\subsection{Representation of Some Poverty Indices}

We may easily find the functions $g$ and $v$ for the most common members of the GPI family (see $[25,28]$ ) in Table 1.

Where

$$
g_{s}(y)=\left\{2\left[\left(1-\frac{G(y)}{G(Z)}\right)\left(\frac{Z-y}{Z}\right)-\left(\frac{G(y)}{G(Z)}\right)\left(\frac{J_{s}(G)}{G(Z)}\right)\right]+K_{s}(G)\right\} \mathbb{I}_{(y \leq Z)},
$$

and

$$
v_{s}(y)=-\frac{2}{G(Z)}\left[\left(\frac{Z-y}{Z}\right)+\frac{J_{s}(G)}{G(Z)}\right] \mathbb{I}_{(y \leq Z)}
$$

with

$$
\begin{gathered}
J_{s}(G)=2 \int_{0}^{G(Z)}\left(1-\frac{s}{G(Z)}\right)\left(\frac{Z-G^{-1}(s)}{Z}\right) \mathrm{d} s, \\
K_{s}(G)=2\left(1-\frac{1}{Z G(Z)} \int_{0}^{G(Z)} G^{-1}(s) \mathrm{d} s\right)+\frac{J_{s}(G)}{G(Z)} .
\end{gathered}
$$

And

$$
\begin{gathered}
g_{k}(y)=\left\{(k+1)\left[\left(1-\frac{G(y)}{G(Z)}\right)^{k}\left(\frac{Z-y}{Z}\right)-\frac{J_{k}(G)}{G(Z)}\left(\frac{G(y)}{G(Z)}\right)^{k}\right]+K_{k}(G)\right\} \mathbb{I}_{(y \leq Z)}, \\
v_{k}(y)=-\frac{k(k+1)}{G(Z)}\left[\left(1-\frac{G(y)}{G(Z)}\right)^{k-1}\left(\frac{Z-y}{Z}\right)+\frac{J_{k}(G)}{G(Z)}\left(\frac{G(y)}{G(Z)}\right)^{k-1}\right] \mathbb{I}_{(y \leq Z)}
\end{gathered}
$$


where

$$
J_{k}(G)=(k+1) \int_{0}^{G(Z)}\left(1-\frac{s}{G(Z)}\right)^{k}\left(\frac{Z-G^{-1}(s)}{Z}\right) \mathrm{d} s,
$$

and

$$
\begin{aligned}
K_{k}(G)= & \frac{k(k+1)}{G(Z)} \int_{0}^{G(Z)}\left(1-\frac{s}{G(Z)}\right)^{k-1}\left(\frac{Z-G^{-1}(s)}{Z}\right) \mathrm{d} s \\
& +\frac{J_{k}(G)}{G(Z)} .
\end{aligned}
$$

Notice that the functions are indexed by $k$ for the Kakwani measure. For the FGT measure of index $\alpha$, we have that $v=0$ and

$$
g(x)=\max (0,(Z-x) / Z)^{\alpha} .
$$

\subsection{Datadriven Applications and Variance Computations}

\subsubsection{Variance Computations for Senegalese Data}

We apply our results to Senegalese data. We do not really have longitudinal data. So we have constructed pseudo-panel data of size $n=116$, from two surveys: ESAM II conducted from 2001 to 2002 and EPS from 2005 to 2006 . We get two series $X^{1}$ and $X^{2}$. We present below the values of $\Gamma_{I}(1,2)$ denoted here $\gamma(1)$, $\Gamma_{J}(1,2)$ denoted here $\gamma(2)$ and $\Gamma(1,2)$ denoted here $\gamma(3)$.

When constructing pseudo-panel data, we get small sizes like $n=116$. We use these sizes to compute the asymptotic variances in our results by mean of nonparametric methods. In real contexts, we should use high sizes comparable to those of the real databases, that is around ten thousands, like in the Senegalese case. Nevertheless, we back on medium sizes, for instance $n=696$, which give very accurate confidence intervals. We use here the abreviations are given in Table 2.

The obtained confidence intervals are described in
Tables 3 to 10, in Subsection 5.2. Before we present the outcomes, let us say some words on the packages. We provide different $\mathrm{R}$ script files at:

http://www.ufrsat.org/lerstad/resources/mergslo01.zip

The user should already have his data in two files data1.txt and data2.txt. The first script file named after gamma_mergslo1.dat provides the values of $\gamma(1)$, $\gamma(2)$ and $\gamma(3)$ for the FGT measure for $\alpha=0,1,2$ and for the six inequality measures used here. The second script file named as gamma_mergslo2.dat performs

Table 1. Specific functions of the poverty measures.

\begin{tabular}{ccc}
\hline Mesure & $g$ & $v$ \\
\hline Shorrocks & $2(1-G(y))\left(\frac{Z-y}{Z}\right) \mathbb{I}_{(y \leq z)}$ & $-2\left(\frac{Z-y}{Z}\right) \mathbb{I}_{(y \leq Z)}$ \\
Thon & $2(1-G(y))\left(\frac{Z-y}{Z}\right) \mathbb{I}_{(y \leq Z)}$ & $-2\left(\frac{Z-y}{Z}\right) \mathbb{I}_{(y \leq Z)}$ \\
Sen & $g_{s}$ & $v_{s}$ \\
Kakwani & $g_{k}$ & $v_{k}$ \\
\hline
\end{tabular}

Table 2. Notation of each measure.

\begin{tabular}{cc}
\hline Notations & Indices \\
\hline GE $(\alpha), \alpha=0.5,2,3$ & Generalized Entropy with parameter $\alpha$ \\
THEIL & Theil \\
MLD & Mean Logarithmic Deviation \\
$\operatorname{ATK}(\alpha), \alpha=0.5,-0.5$ & Atkinson with parameter $\alpha$ \\
CHAMP & Champernowne \\
SHOR & Shorrocks \\
SEN & Sen \\
$\operatorname{KAK}(k), k=1,2$ & Kakwani with parameter $k$ \\
$\operatorname{FGT}(\alpha), \alpha=0,1,2$ & Foster-Greer-Thorbecke with parameter $\alpha$ \\
\hline
\end{tabular}

Table 3. Variations of the inequality indices.

\begin{tabular}{cccc}
\hline Indice & $\Delta I(1,2)$ & $\Gamma_{I}(1,2)$ & $C I_{95 \%}(\Delta I(1,2))$ \\
\hline GE $(0.5)$ & -0.04025832 & 0.01770106 & {$[-0.05588673 ;-0.03611789]$} \\
GE (2) & -0.06408679 & 0.07224733 & {$[-0.09545863 ;-0.05552007]$} \\
GE (3) & -0.1008038 & 0.1205114 & {$[-0.1495352 ;-0.09795348]$} \\
THEIL & -0.04569319 & 0.02223474 & {$[-0.0635651 ;-0.04140879]$} \\
MLD & -0.03645671 & 0.01523784 & {$[-0.05085476 ;-0.03251291]$} \\
ATK (0.5) & -0.01976068 & 0.004225092 & {$[-0.02742201 ;-0.01776374]$} \\
ATK $(-0.5)$ & -0.04423886 & 0.02212773 & {$[-0.06159485 ;-0.03949192]$} \\
CHAMP & -0.03421829 & 0.01283687 & {$[-0.04734396 ;-0.03050904]$} \\
\hline
\end{tabular}


Table 4. Variations of the poverty indices.

\begin{tabular}{cccc}
\hline Ratio & $\Delta J(1,2)$ & $\Gamma_{J}(1,2)$ & $C I_{95 \%}(\Delta J(1,2))$ \\
\hline SHOR & -0.03024621 & 0.02353406 & {$[-0.04264967 ;-0.01985518]$} \\
KAK (1) & -0.02108905 & 0.01097123 & {$[-0.02982085 ;-0.01425729]$} \\
KAK (2) & -0.02055594 & 0.01007820 & {$[-0.02961271 ;-0.01469601]$} \\
FGT (0) & -0.05977098 & 0.3170756 & {$[-0.09355847 ;-0.009889805]$} \\
FGT (1) & -0.01859332 & 0.00922992 & {$[-0.02620413 ;-0.01192899]$} \\
FGT (2) & -0.00432289 & 0.0008381113 & {$[-0.007194404 ;-0.002892781]$} \\
\hline
\end{tabular}

Table 5. Ratio of the variations with Shorrocks measure.

\begin{tabular}{ccccc}
\hline Ratio & $R(1,2)$ & $\Gamma_{I J}(1,2)$ & $\Gamma(1,2)$ & $C I_{95 \%}(R(1,2))$ \\
\hline SHOR/GE $(0.5)$ & 0.7513034 & 0.005477263 & 15.60737 & {$[0.3858608 ; 0.9728719]$} \\
SHOR/GE (2) & 0.471957 & 0.006487665 & 8.157275 & {$[0.2018082 ; 0.6261873]$} \\
SHOR/GE (3) & 0.3000503 & 0.009018111 & 2.851175 & {$[0.1271085 ; 0.3780043]$} \\
SHOR/THEIL & 0.6619413 & 0.005642781 & 12.36007 & {$[0.3342390 ; 0.8566255]$} \\
SHOR/MLD & 0.8296473 & 0.8296473 & 18.77303 & {$[0.4278509 ; 1.071647]$} \\
SHOR/ATK (0.5) & 1.530626 & 0.002695030 & 64.49043 & {$[0.7866646 ; 1.979908]$} \\
SHOR/ATK (-0.5) & 0.6837023 & 0.007288597 & 12.21780 & {$[0.555278 ; 1.395697]$} \\
SHOR/CHAMP & 0.8839194 & 0.005165236 & 20.86647 & {$[0.4634852 ; 1.142229]$} \\
\hline
\end{tabular}

Table 6. Ratio of the variations with Sen measure.

\begin{tabular}{ccccc}
\hline Ratio & $R(1,2)$ & $\Gamma_{I J}(1,2)$ & $\Gamma(1,2)$ & $C I_{95 \%}(R(1,2))$ \\
\hline SEN/GE (0.5) & 0.3290702 & 0.003112166 & 7.754599 & {$[0.272201 ; 0.6859714]$} \\
SEN/GE (2) & 0.3290702 & 0.003512353 & 4.013294 & {$[0.1431155 ; 0.4407834]$} \\
SEN/GE (3) & 0.2092089 & 0.005939808 & 1.354192 & {$[0.0916464 ; 0.2645570]$} \\
SEN/THEIL & 0.461536 & 0.003364929 & 6.035583 & {$[0.237376 ; 0.6024165]$} \\
SEN/MLD & 0.5784683 & 0.002968939 & 9.506736 & {$[0.2996504 ; 0.7577893]$} \\
SEN/ATK (0.5) & 1.067223 & 0.001542060 & 31.99108 & {$[0.555278 ; 1.395697]$} \\
SEN/ATK (-0.5) & 0.4360427 & 0.003368434 & 6.534366 & {$[0.2461303 ; 0.625955]$} \\
SEN/CHAMP & 0.6163094 & 0.003038844 & 10.33521 & {$[0.3273292 ; 0.8050137]$} \\
\hline
\end{tabular}

Table 7. Ratio of the variations with Kakwani (2) measure.

\begin{tabular}{ccccc}
\hline Ratio & $R(1,2)$ & $\Gamma_{I J}(1,2)$ & $\Gamma(1,2)$ & $C I_{95 \%}(R(1,2))$ \\
\hline KAK (2)/GE (0.5) & 0.510601 & 0.002574653 & 7.443462 & {$[0.2788993 ; 0.6842854]$} \\
KAK (2)/GE (2) & 0.3207516 & 0.008486058 & 2.93814 & {$[0.1661299 ; 0.4208233]$} \\
KAK (2)/GE (3) & 0.2039203 & 0.005185377 & 1.276858 & {$[0.09508295 ; 0.2629838]$} \\
KAK (2)/THEIL & 0.4498688 & 0.002906321 & 5.72986 & {$[0.2442552 ; 0.5999303]$} \\
KAK (2)/MLD & 0.5638451 & 0.002365820 & 9.220372 & {$[0.3058926 ; 0.7570787]$} \\
KAK (2)/ATK (0.5) & 1.040245 & 0.001292464 & 30.63183 & {$[0.5694048 ; 1.391776]$} \\
KAK (2)/ATK (-0.5) & 0.4646579 & 0.001933209 & 6.672792 & {$[0.2464103 ; 0.630237]$} \\
KAK (2)/CHAMP & 0.6007296 & 0.002781442 & 9.709634 & {$[0.3376321 ; 0.8006341]$} \\
\hline
\end{tabular}


Table 8. Ratio of the variations with FGT (1) measure.

\begin{tabular}{ccccc}
\hline Ratio & $R(1,2)$ & $\Gamma_{I J}(1,2)$ & $\Gamma(1,2)$ & $C I_{95 \%}(R(1,2))$ \\
\hline FGT (1)/GE (0.5) & 0.4618504 & 0.003359959 & 6.109622 & {$[0.2308332 ; 0.5981059]$} \\
FGT (1)/GE (2) & 0.29901272 & 0.004159761 & 3.140289 & {$[0.2316082 ; 0.4949175]$} \\
FGT (1)/GE (3) & 0.1844506 & 0.005815332 & 1.100702 & {$[0.0761356 ; 0.2320249]$} \\
FGT (1)/THEIL & 0.4069167 & 0.003487018 & 4.824886 & {$[0.2000723 ; 0.5264534]$} \\
FGT (1)/MLD & 0.5100109 & 0.003329621 & 7.371324 & {$[0.2557003 ; 0.6591174]$} \\
FGT (1)/ATK (0.5) & 0.9409253 & 0.001652060 & 25.25488 & {$[0.4705622 ; 1.217276]$} \\
FGT (1)/ATK (-0.5) & 0.4202938 & 0.004429351 & 4.81098 & {$[0.2142764 ; 0.5401868]$} \\
FGT (1)/CHAMP & 0.5433737 & 0.003126249 & 8.218207 & {$[0.2768286 ; 0.7027897]$} \\
\hline
\end{tabular}

Table 9. Ratio of the variations with FGT (0) measure.

\begin{tabular}{ccccc}
\hline Ratio & $R(1,2)$ & $\Gamma_{\nu J}(1,2)$ & $\Gamma(1,2)$ & $C I_{95 \%}(R(1,2))$ \\
\hline FGT $(0) /$ GE $(0.5)$ & 1.484686 & 1.484686 & 192.9616 & {$[0.09236428 ; 2.156398]$} \\
FGT (0)/GE (2) & 0.9326567 & 0.02159780 & 82.69382 & {$[0.009587167 ; 1.360782]$} \\
FGT (0)/GE (3) & 0.5929437 & 0.03215672 & 31.62072 & {$[0.0002219161 ; 0.8357621]$} \\
FGT (0)/THEIL & 1.308094 & 0.01626234 & 149.7108 & {$[0.07643712 ; 1.894496]$} \\
FGT (0)/MLD & 1.639505 & 0.01332770 & 236.7108 & {$[0.09833456 ; 2.383401]$} \\
FGT (0)/ATK (0.5) & 3.024743 & 0.00717539 & 799.837 & {$[0.1882737 ; 4.390527]$} \\
FGT (0)/ATK (-0.5) & 1.351097 & 0.01606948 & 160.4669 & {$[0.08224307 ; 1.964480]$} \\
FGT (0)/CHAMP & 1.746755 & 0.01248913 & 266.9863 & {$[0.1148277 ; 2.542700]$} \\
\hline
\end{tabular}

Table 10. Ratio of the variations with FGT (2) measure.

\begin{tabular}{ccccc}
\hline Ratio & $R(1,2)$ & $\Gamma_{I J}(1,2)$ & $\Gamma(1,2)$ & $C I_{95 \%}(R(1,2))$ \\
\hline FGT (2)/GE (0.5) & 0.1073788 & 0.000974483 & 0.5139224 & {$[0.05637792 ; 0.1628977]$} \\
FGT (2)/GE (2) & 0.06745369 & 0.001055690 & 0.2494247 & {$[0.02970793 ; 0.103916]$} \\
FGT (2)/GE (3) & 0.0428842 & 0.001371335 & 0.09271563 & {$[0.01813633 ; 0.06338001]$} \\
FGT (2)/THEIL & 0.09460689 & 0.0009653898 & 0.4092489 & {$[0.04856479 ; 0.1436198]$} \\
FGT (2)/MLD & 0.118576 & 0.001013111 & 0.6110173 & {$[0.06292282 ; 0.1790699]$} \\
FGT (2)/ATK (0.5) & 0.2187623 & 0.0004795731 & 2.126811 & {$[0.1148914 ; 0.3315849]$} \\
FGT (2)/ATK (-0.5) & 0.09771703 & 0.001424631 & 0.3939442 & {$[0.05315702 ; 0.1464178]$} \\
FGT (2)/CHAMP & 0.1263327 & 0.000954164 & 0.6848654 & {$[0.0680842 ; 0.1910499]$} \\
\hline
\end{tabular}

the same for the Shorrocks measure. Lastly, gamma mergslo3.dat concerns the kakwani measures. Unless the user uploads new data1.txt and data2.txt files, the outcomes should be the same as those presented in the Appendix.

\subsubsection{Analysis}

First of all, we find in Tables $\mathbf{3}$ and $\mathbf{4}$ in the appendix 5 that at an asymptotical level, all our inequality measures and poverty indices used here have decreased. When inspecting the asymptotic variance, we see in Table 4 that for the poverty indice, the FGT and the Kakwani classes respectively for $\alpha=1, \alpha=2$ and $k=1, k=2$ have the minimum variance, specially for $\alpha=2$ and $k$ $=2$. This advocates for the use of the Kakwani and the FGT measures for poverty reduction evaluation. As for the inequality approach in Table 3, it seems that Atkinson measure ATK (0.5) has the minimum variance and then is recommended.

As for the ratio of the poverty index over the inequality 
Table 11. Dependence of over $\mathbf{5 0 \%}$.

\begin{tabular}{cccc}
\hline Couples & (KAK (2), GE (0.5)) & (KAK (2), MLD) & (FGT (1), CHAMP) \\
Dependences (\%) & 51.06 & 51.06 & 54.33 \\
\hline Couples & (SEN, MLD) & (KAK (2), CHAMP) & (SEN, CHAMP) \\
Dependences (\%) & 57.84 & 60.07 & 61.63 \\
\hline Couples & (SHOR, THEIL) & (SHOR, ATK (-0.5)) & 75.13 \\
Dependences (\%) & 66.19 & 68.37 & $($ SHOR, GE (0.5)) \\
\hline Couples & $($ SHOR, MLD) & (SHOR, CHAMP) & 153.06 \\
\hline
\end{tabular}

measure, we have a dependence of over $50 \%$ for the following couples in Table 11, that we can find in Tables 5 to 8 .

The maximum ratio 3.024 is attained for FGT $(0)$ and Atkinson (0.5). Based on these data, and on the confidence intervals in Table 9, we would report at least of $46.43 \%$ for these two measures and conclude that the gain over poverty in Senegal between these two periods is significally pro-poor. We would have worked with all couples with a ratio over $50 \%$ to have the same conclusion.

The present analysis should be developped in a separated paper research since this one was devoted to a theoritical basis. We plan to apply at a regional basis, that is for the countries of the UEMOA in West Africa.

\section{Conclusion}

We have been able to compute confidence intervals for the ratio of variations for the poverty and the inequality indices. The results enabled us to cheek whether the growth is pro or against poor in Senegal from 2002 to 2006. It always remains to undertake large scale data driven applications at a regional level, precisely in the UEMOA African area. We used in this paper a Theil-like family of inequality measures that does not include the celebrated and important Gini index. Moreover other the Theil-like families exist. It would be interesting to have the same theory developed here using the Gini index and other families as well. We plan to do it in a very close future.

\section{Acknowledgements}

We express our thanks to the Ministère de l'Enseignement Supérieur et de la Recherche for financial support under a FIRST grant 2013-2014.

\section{REFERENCES}

[1] G. S. Lo, S. T. Sall and C. T. Seck, "Une Théorie Asymptotique des Indicateurs de Pauvreté," Comptes Ren- dus Mathématiques de l'Académie des Sciences. Mathematical Reports of the Academy of Canada, Vol. 31, No. 2, 2009, pp. 45-52.

[2] G. S. Lo, "The Generalized Poverty Index," Far East Journal of Theoretical Statistics, Vol. 42, No. 1, 2013, pp. 1-22.

[3] B. Zheng, “Aggregate Poverty Measures," Journal of Economic Surveys, Vol. 11, No. 2, 1997, pp. 123-162. doi:10.1111/1467-6419.00028

[4] K. A. Sen, "Poverty: An Ordinal Approach to Measurement," Econometrica, Vol. 44, No. 2, 1976, pp. 219-231. doi: $10.2307 / 1912718$

[5] N. Kakwani, "On a Class of Poverty Measures," Econometrica, Vol. 48, No. 2, 1980, pp. 437-446. doi:10.2307/1911106

[6] A. Shorrocks, "Revisiting the Sen Poverty Index," Econometrica, Vol. 63, No. 5, 1995, pp. 1225-1230. doi: $10.2307 / 2171728$

[7] S. Clark, R. Hemming and D. Ulph, "On Indices for the Measurement of Poverty," Economic Journal, Vol. 91, 1981, pp. 525-526. doi:10.2307/2232600

[8] J. E. Foster, J. Greer and E. Thorbecke, "A Class of Decomposable Poverty Measures," Econometrica, Vol. 52, No. 3, 1984, pp. 761-766. doi:10.2307/1913475

[9] S. T. Sall and G. S. Lo, "The Asymptotic Theory of the Poverty Intensity in View of Extreme Values Theory for Two Simple Cases," Afrika Statistika, Vol. 2, No. 1, 2007, pp. 41-55.

[10] S. T. Sall and G. S. Lo, "Uniform Weak Convergence of the Time-Dependent Poverty Measure for Continuous Longitudinal Data," Brazilian Journal of Probability and Statistics, Vol. 24, No. 3, 2010, pp. 457-467. doi:10.1214/08-BJPS101

[11] F. A. Cowell, "Theil, Inequality and the Structure of Income Distribution," London School of Economics and Political Sciences, London, 2003. doi:10.1016/0014-2921(80)90051-3

[12] H. Theil, "Economics and Information Theory," North Holland, Amsterdam, 1967.

[13] F. A. Cowell, "Generalized Entropy and the Measurement of Distributional Change," European Economic Review, Vol. 13, No. 1, 1980, pp. 147-159.

[14] A. B. Atkinson, "On the Measurement of Inequality," Jour- 
nal of Economic Theory, Vol. 2, No. 3, 1970, pp. 244263. doi:10.1016/0022-0531(70)90039-6

[15] D. G. Champernowne and F. A. Cowell, "Economic Inequality and Income Distribution," Cambridge University Press, Cambridge, 1998.

[16] S. Kolm, "Unequal Inequalities I," Journal of Economic Theory, Vol. 12, No. 3, 1976, pp. 416-442. doi:10.1016/0022-0531(76)90037-5

[17] A. K. Sen, “On Economic Inequality," Clarendon Press, Oxford, 1973. doi:10.1093/0198281935.001.0001

[18] J. C. Harsanyi, "Cardinal Utility in Welfare Economics of Concentration," Journal of the Royal Statistical Society, Series A, Vol. 123, 1953, pp. 423-434.

[19] J. C. Harsanyi, "Cardinal Welfare, Individualistic Ethics and Interpersonal Comparisons of Utility," Journal of Political Economy, Vol. 63, No. 4, 1955, pp. 309-321. doi:10.1086/257678

[20] M. Rothschild and J. E. Stiglitz, "Some Further Results on the Measurement of Inequality," Journal of Economic Theory, Vol. 6, 1973, pp. 188-203. doi:10.1016/0022-0531(73)90034-3

[21] L. S. Temkin, "Inequality," Oxford University Press, Oxford, 1993.
[22] S. Kullback, "Inference Theory and Statistics," John Wiley, New York, 1959.

[23] F. A. Cowell, "On the Structure of Additive in Equality Measures," Review of Economic Studies, Vol. 47, No. 3, 1980, pp. 521-531. doi:10.2307/2297303

[24] F. A. Cowell, "Measurement of Inequality," In: A. B. Atkinson and F. Bourguignon, Eds., Handbook of Income Distribution, 2000, pp. 87-166. doi:10.1016/S1574-0056(00)80005-6

[25] G. S. Lo and S. T. Sall, "Asymptotic Representation Theorems for Poverty Indices," Afrika Statistika, Vol. 5, 1996, pp. 238-244.

[26] A. W. Van der Vaart and J. A. Wellner, "Weak Convergence and Empirical Processes: With Applications to Statistics," Springer-Verlag, New York, 1996. doi:10.1007/978-1-4757-2545-2

[27] G. R. Shorack and J. A. Wellner, "Empirical Processes with Applications to Statistics," Wiley-Interscience, New York, 1986.

[28] G. S. Lo, "A Simple Note on Some Empirical Stochastic Process as a Tool in Uniform L-Statistics Weak Laws," Afrika Statistika, Vol. 5, No. 7, 2010, pp. 245-251. 


\section{Appendix}

\section{Proofs of the Theorems}

\section{Proof of Theorem 2.}

By using the delta-method, we have for all $i \in\{1,2\}$ :

$$
\begin{aligned}
\sqrt{n}\left\{h_{1}\left(\mu_{n}(i)\right)-h_{1}(\mu(i))\right\} & =h_{1}^{\prime}(\mu(i)) \sqrt{n}\left(\mu_{n}(i)-\mu(i)\right)+o_{p}(1)=h_{1}^{\prime}(\mu(i)) \frac{1}{\sqrt{n}} \sum_{j=1}^{n}\left(X_{j}^{i}-\mathbb{E}\left(X_{j}^{i}\right)\right)+o_{p}(1) \\
& =h_{1}^{\prime}(\mu(i)) \frac{1}{\sqrt{n}} \sum_{j=1}^{n}\left(\tilde{f}_{i}\left(U_{j}, V_{j}\right)-\mathbb{P}_{(U, V)}\left(\tilde{f}_{i}\right)\right)+o_{p}(1)=h_{1}^{\prime}(\mu(i)) \mathbb{G}_{n}\left(\tilde{f}_{i}\right)+o_{p}(1) .
\end{aligned}
$$

Then

$$
\sqrt{n}\left\{h_{1}\left(\mu_{n}(i)\right)-h_{1}(\mu(i))\right\}=\mathbb{G}_{n}\left(h_{1}^{\prime}(\mu(i)) \tilde{f}_{i}\right)+o_{p}(1) .
$$

Similarly, we have

$$
\sqrt{n}\left\{h_{2}\left(\mu_{n}(i)\right)-h_{2}(\mu(i))\right\}=\mathbb{G}_{n}\left(h_{2}^{\prime}(\mu(i)) \tilde{f}_{i}\right)+o_{p}(1) .
$$

From this and (3.1), we have

$$
\sqrt{n}\left\{B_{n}(i)-B(i)\right\}=\frac{1}{\sqrt{n}} \sum_{j=1}^{n}\left(h\left(X_{j}^{i}\right)-\mathbb{E}\left(h\left(X_{j}^{i}\right)\right)\right)=\frac{1}{\sqrt{n}} \sum_{j=1}^{n}\left(f_{i, h}\left(U_{j}, V_{j}\right)-\mathbb{P}_{(U, V)}\left(f_{i, h}\right)\right) ;
$$

and then

$$
\sqrt{n}\left\{B_{n}(i)-B(i)\right\}=\mathbb{G}_{n}\left(f_{i, h}\right) .
$$

Further

$$
\begin{aligned}
\sqrt{n}\left\{I_{n}(i)-I(i)\right\} & =\sqrt{n}\left\{\tau\left(\frac{B_{n}(i)}{h_{1}\left(\mu_{n}(i)\right)}-h_{2}\left(\mu_{n}(i)\right)\right)-\tau\left(\frac{B(i)}{h_{1}(\mu(i))}+h_{2}(\mu(i))\right)\right\} \\
& =K_{i} \sqrt{n}\left\{\frac{B_{n}(i)}{h_{1}\left(\mu_{n}(i)\right)}-h_{2}\left(\mu_{n}(i)\right)-\frac{B(i)}{h_{1}(\mu(i))}+h_{2}(\mu(i))\right\}+o_{p}(1) .
\end{aligned}
$$

But

$$
\begin{aligned}
& \sqrt{n}\left\{\frac{B_{n}(i)}{h_{1}\left(\mu_{n}(i)\right)}-h_{2}\left(\mu_{n}(i)\right)-\frac{B(i)}{h_{1}(\mu(i))}+h_{2}(\mu(i))\right\} \\
& =\frac{\sqrt{n}\left\{B_{n}(i)-B(i)\right\}}{h_{1}\left(\mu_{n}(i)\right)}-\left(\frac{B(i) h_{1}^{\prime}(\mu(i))}{h_{1}\left(\mu(i) h_{1}\left(\mu_{n}(i)\right)\right)}+h_{2}^{\prime}(\mu(i))\right) \sqrt{n}\left\{\mu_{n}(i)-\mu(i)\right\}+o_{p}(1) \\
& =\frac{1}{h_{1}\left(\mu_{n}(i)\right)} \mathbb{G}_{n}\left(f_{i, h}\right)-\left(\frac{B(i) h_{1}^{\prime}(\mu(i))}{h_{1}\left(\mu(i) h_{1}\left(\mu_{n}(i)\right)\right)}+h_{2}^{\prime}(\mu(i))\right) \mathbb{G}_{n}\left(\tilde{f}_{i}\right)+o_{p}(1) \\
& =\mathbb{G}_{n}\left(\frac{1}{h_{1}(\mu(i))} f_{i, h}-\left(\frac{B(i) h_{1}^{\prime}(\mu(i))}{h_{1}^{2}(\mu(i))}+h_{2}^{\prime}(\mu(i))\right) \tilde{f}_{i}\right)+o_{p}(1) .
\end{aligned}
$$

Thus

$$
\sqrt{n}\left\{I_{n}(i)-I(i)\right\}=K_{i} \mathbb{G}_{n}\left(\frac{1}{h_{1}(\mu(i))} f_{i, h}-\left(\frac{B(i) h_{1}^{\prime}(\mu(i))}{h_{1}^{2}(\mu(i))}+h_{2}^{\prime}(\mu(i))\right) \tilde{f}_{i}\right)+o_{p}(1),
$$

that is

$$
\sqrt{n}\left\{I_{n}(i)-I(i)\right\}=\mathbb{G}_{n}\left(F_{i, I}^{\star}\right)+o_{p}(1) .
$$


Finally using the linearity of the FEP, we get

$$
\begin{aligned}
\sqrt{n}\left\{\Delta I_{n}(1,2)-\Delta I(1,2)\right\} & =\sqrt{n}\left\{I_{n}(2)-I(2)\right\}-\sqrt{n}\left\{I_{n}(1)-I(1)\right\} \\
& =\mathbb{G}_{n}\left(F_{2, I}^{\star}\right)-\mathbb{G}_{n}\left(F_{1, I}^{\star}\right)+o_{p}(1)=\mathbb{G}_{n}\left(F_{2, I}^{\star}-F_{1, I}^{\star}\right)+o_{p}(1)
\end{aligned}
$$

and conclude by

$$
\sqrt{n}\left\{\Delta I_{n}(1,2)-\Delta I(1,2)\right\}=\mathbb{G}_{n}\left(F_{I}^{\star}\right)+o_{p}(1)
$$

and

$$
\Gamma_{I}(1,2)=\mathbb{E}\left(\mathbb{G}\left(F_{I}^{\star}\right)^{2}\right)=\int_{D^{2}}\left(F_{I}^{\star}(u, v)-\mathbb{P}_{(U, V)}\left(F_{I}^{\star}\right)\right)^{2} \mathrm{~d} C(u, v) .
$$

Proof of Theorem 3. We have

$$
J_{n}(i)=\frac{1}{n} \sum_{j=1}^{n} c\left(G_{n}^{i}\left(X_{j, n}^{i}\right)\right) q_{i}\left(X_{j, n}^{i}\right)
$$

and then

$$
\begin{aligned}
\sqrt{n}\left\{J_{n}(i)-J(i)\right\}= & \frac{1}{\sqrt{n}} \sum_{j=1}^{n}\left(g_{i}\left(X_{j, n}^{i}\right)-\mathbb{E} g_{i}\left(X_{j, n}^{i}\right)\right)+\int_{D} \alpha_{n}(s) v_{i}(s) \mathrm{d} s+o_{p}(1) \\
= & \frac{1}{\sqrt{n}} \sum_{j=1}^{n}\left(g_{i} \circ G_{i}^{-1} \circ \Pi_{i}\left(U_{j, n}, V_{j, n}\right)-\mathbb{E} g_{i} \circ G_{i}^{-1} \circ \Pi_{i}\left(U_{j, n}, V_{j, n}\right)\right) \\
& +\int_{D} \frac{1}{\sqrt{n}} \sum_{j=1}^{n}\left(\Pi_{i}\left(\mathbb{I}_{(0, s)}\left(U_{j, n}\right), \mathbb{I}_{(0, s)}\left(V_{j, n}\right)\right)-\mathbb{E}_{i}\left(\mathbb{I}_{(0, s)}\left(U_{j, n}\right), \mathbb{I}_{(0, s)}\left(V_{j, n}\right)\right)\right) v_{i}(s) \mathrm{d} s+o_{p}(1) \\
= & \frac{1}{\sqrt{n}} \sum_{j=1}^{n}\left(F_{i, J}^{\star}\left(U_{j, n}, V_{j, n}\right)-\mathbb{P}_{(U, V)}\left(F_{i, J}^{\star}\right)\right)+\int_{D} \frac{1}{\sqrt{n}} \sum_{j=1}^{n}\left(f_{i, s}\left(U_{j, n}, V_{j, n}\right)-\mathbb{P}_{(U, V)}\left(f_{i, s}\right)\right) v_{i}(s) \mathrm{d} s+o_{p}(1) .
\end{aligned}
$$

We arrive at

$$
\sqrt{n}\left\{J_{n}(i)-J(i)\right\}=\mathbb{G}_{n}\left(F_{i, J}^{\star}\right)+\int_{D} \mathbb{G}_{n}\left(f_{i, s}\right) v_{i}(s) \mathrm{d} s+o_{p}(1) .
$$

We get the variation of $J_{n}$ between to instants $i=1$ and $i=2$ as follows

$$
\begin{aligned}
\sqrt{n}\left\{\Delta J_{n}(1,2)-\Delta J(1,2)\right\} & =\sqrt{n}\left\{J_{n}(2)-J(2)\right\}-\sqrt{n}\left\{J_{n}(1)-J(1)\right\} \\
& =\mathbb{G}_{n}\left(F_{2, J}^{\star}-F_{1, J}^{\star}\right)+\int_{D}\left(\mathbb{G}_{n}\left(f_{2, s}\right) v_{2}(s)-\mathbb{G}_{n}\left(f_{1, s}\right) v_{1}(s)\right) \mathrm{d} s+o_{p}(1) .
\end{aligned}
$$

This leads to

$$
\sqrt{n}\left\{\Delta J_{n}(1,2)-\Delta J(1,2)\right\}=\mathbb{G}_{n}\left(F_{J}^{\star}\right)+\int_{D}\left(\mathbb{G}_{n}\left(f_{2, s}\right) v_{2}(s)-\mathbb{G}_{n}\left(f_{1, s}\right) v_{1}(s)\right) \mathrm{d} s+o_{p}(1) .
$$

The proof will be complete with the expression of $\Gamma_{J}(1,2)$. We have

$$
\begin{aligned}
\Gamma_{J}(1,2)= & \mathbb{E}\left(\left(\mathbb{G}\left(F_{J}^{\star}\right)+\int_{D}\left(\mathbb{G}\left(f_{2, s}\right) v_{2}(s)-\mathbb{G}\left(f_{1, s}\right) v_{1}(s)\right) \mathrm{d} s\right)^{2}\right) \\
= & \mathbb{E}\left(\mathbb{G}\left(F_{J}^{\star}\right)^{2}\right)+\mathbb{E}\left(\left(\int_{D}\left(\mathbb{G}\left(f_{2, s}\right) v_{2}(s)-\mathbb{G}\left(f_{1, s}\right) v_{1}(s)\right) \mathrm{d} s\right)^{2}\right) \\
& +2 \mathbb{E}\left(\mathbb{G}\left(F_{J}^{\star}\right) \int_{D}\left(\mathbb{G}\left(f_{2, s}\right) v_{2}(s)-\mathbb{G}\left(f_{1, s}\right) v_{1}(s)\right) \mathrm{d} s\right) . \\
\equiv & \Gamma_{1}(1,2)+\Gamma_{2}(1,2)+2 \Gamma_{3}(1,2) .
\end{aligned}
$$

Let us compute these three numbers. First consider,

$$
\Gamma_{1}(1,2)=\mathbb{E}\left(\mathbb{G}\left(F_{J}^{\star}\right)^{2}\right)=\int_{D^{2}}\left(F_{J}^{\star}(u, v)-\mathbb{P}_{(U, V)}\left(F_{J}^{\star}\right)\right)^{2} d C(u, v) .
$$

Secondly, compute

$$
\Gamma_{2}(1,2)=\mathbb{E}\left(\left(\int_{D}\left(v_{2}(s) \mathbb{G}\left(f_{2, s}\right)-v_{1}(s) \mathbb{G}\left(f_{1, s}\right)\right) \mathrm{d} s\right)^{2}\right)
$$


By developing and applying Fubini to this term, we get

$$
\begin{aligned}
\Gamma_{2}(1,2)= & \int_{D^{2}} v_{2}(s) v_{2}(t) \mathbb{E}\left(\mathbb{G}\left(f_{2, s}\right) \mathbb{G}\left(f_{2, t}\right)\right) \mathrm{d} s \mathrm{~d} t-\int_{D^{2}} v_{2}(s) v_{1}(t) \mathbb{E}\left(\mathbb{G}\left(f_{2, s}\right) \mathbb{G}\left(f_{1, t}\right)\right) \mathrm{d} s \mathrm{~d} t \\
& -\int_{D^{2}} v_{1}(s) v_{2}(t) \mathbb{E}\left(\mathbb{G}\left(f_{1, s}\right) \mathbb{G}\left(f_{2, t}\right)\right) \mathrm{d} s \mathrm{~d} t+\int_{D^{2}} v_{1}(s) v_{1}(t) \mathbb{E}\left(\mathbb{G}\left(f_{1, s}\right) \mathbb{G}\left(f_{1, t}\right)\right) \mathrm{d} s \mathrm{~d} t
\end{aligned}
$$

or

$$
\begin{aligned}
\mathbb{E}\left(\mathbb{G}\left(f_{2, s}\right) \mathbb{G}\left(f_{2, t}\right)\right) & =\mathbb{E}\left(\left(\mathbb{I}_{(0, s)}(V)-s\right)\left(\mathbb{I}_{(0, t)}(V)-t\right)\right)=\min (s, t)-s t \\
\mathbb{E}\left(\mathbb{G}\left(f_{2, s}\right) \mathbb{G}\left(f_{1, t}\right)\right) & =\mathbb{E}\left(\left(\mathbb{I}_{(0, s)}(V)-s\right)\left(\mathbb{I}_{(0, t)}(U)-t\right)\right)=C(t, s)-s t
\end{aligned}
$$

then

$$
\int_{D^{2}} v_{2}(s) v_{2}(t) \mathbb{E}\left(\mathbb{G}\left(f_{2, s}\right) \mathbb{G}\left(f_{2, t}\right)\right) \mathrm{d} s \mathrm{~d} t=\int_{D^{2}} v_{2}(s) v_{2}(t)(\min (s, t)-s t) \mathrm{d} s \mathrm{~d} t ;
$$

and

$$
\int_{D^{2}} v_{2}(s) v_{2}(t) \mathbb{E}\left(\mathbb{G}\left(f_{2, \mathrm{~s}}\right) \mathbb{G}\left(f_{1, t}\right)\right) \mathrm{d} s \mathrm{~d} t=\int_{D^{2}} v_{2}(s) v_{1}(t)(C(t, s)-s t) \mathrm{d} s \mathrm{~d} t
$$

Similarly we obtain

$$
\begin{aligned}
& \int_{D^{2}} v_{1}(s) v_{2}(t) \mathbb{E}\left(\mathbb{G}\left(f_{1, s}\right) \mathbb{G}\left(f_{2, t}\right)\right) \mathrm{d} s \mathrm{~d} t=\int_{D^{2}} v_{1}(s) v_{2}(t)(C(s, t)-s t) \mathrm{d} s \mathrm{~d} t \\
& \int_{D^{2}} v_{1}(s) v_{1}(t) \mathbb{E}\left(\mathbb{G}\left(f_{1, s}\right) \mathbb{G}\left(f_{1, t}\right)\right) \mathrm{d} s \mathrm{~d} t=\int_{D^{2}} v_{1}(s) v_{1}(t)(\min (s, t)-s t) \mathrm{d} s \mathrm{~d} t
\end{aligned}
$$

But

$$
\int_{D^{2}} v_{1}(t) v_{2}(s)(C(t, s)-s t) \mathrm{d} s \mathrm{~d} t=\int_{D^{2}} v_{1}(s) v_{2}(t)(C(s, t)-s t) \mathrm{d} s \mathrm{~d} t .
$$

By identification, we get

$$
\Gamma_{2}(1,2)=\gamma_{1}-2 \gamma_{2}+\gamma_{3}
$$

and remind that these quantities were defined in Theorem (3). Finally, we have

$$
\begin{aligned}
\Gamma_{3}(1,2)= & \mathbb{E}\left(\mathbb{G}\left(F_{J}^{\star}\right) \int_{D}\left(\mathbb{G}\left(f_{2, s}\right) v_{2}(s)-\mathbb{G}\left(f_{1, s}\right) v_{1}(s)\right) \mathrm{d} s\right) \\
= & \int_{D} v_{2}(s) \mathbb{E}\left(\mathbb{G}\left(F_{J}^{\star}\right) \mathbb{G}\left(f_{2, s}\right)\right) \mathrm{d} s-\int_{D} v_{1}(s) \mathbb{E}\left(\mathbb{G}\left(F_{J}^{\star}\right) \mathbb{G}\left(f_{1, s}\right)\right) \mathrm{d} s \\
= & \int_{D}\left\{v_{2}(s) \int_{(0,1) \times(0, s)} F_{J}^{\star}(u, v) \mathrm{d} C(u, v)-v_{1}(s) \int_{(0, s) \times(0,1)} F_{J}^{\star}(u, v) \mathrm{d} C(u, v)\right\} \mathrm{d} s \\
& -\mathbb{P}_{(U, V)}\left(F_{J}^{\star}\right) \int_{D} s\left(v_{2}(s)-v_{1}(s)\right) \mathrm{d} s .
\end{aligned}
$$

This achieves the proof of Theorem (3).

\section{Proof of Theorem 4.}

By (6) and (10), is clear that

$$
\left(\sqrt{n}\left(\Delta J_{n}(1,2)-\Delta J(1,2)\right), \sqrt{n}\left(\Delta I_{n}(1,2)-\Delta I(1,2)\right)\right)
$$

is asymptotically Gaussian with covariance

$$
\begin{aligned}
\Gamma_{I, J}(1,2) & =\mathbb{E}\left(\mathbb{G}\left(F_{I}^{\star}\right)\left(\mathbb{G}\left(F_{J}^{\star}\right)+\int_{D}\left(v_{2}(s) \mathbb{G}\left(f_{2, s}\right)-v_{1}(s) \mathbb{G}\left(f_{1, s}\right)\right) \mathrm{d} s\right)\right) \\
& =\mathbb{E}\left(\mathbb{G}\left(F_{I}^{\star}\right) \mathbb{G}\left(F_{J}^{\star}\right)\right)+\int_{D} v_{2}(s) \mathbb{E}\left(\mathbb{G}\left(F_{I}^{\star}\right) \mathbb{G}\left(f_{2, s}\right)\right) \mathrm{d} s-\int_{D} v_{1}(x) \mathbb{E}\left(\mathbb{G}\left(F_{I}^{\star}\right) \mathbb{G}\left(f_{1, s}\right)\right) \mathrm{d} s .
\end{aligned}
$$

Then

$$
\begin{aligned}
\Gamma_{I, J}(1,2)= & \mathbb{P}_{(U, V)}\left(\left(F_{I}^{\star}-\mathbb{P}_{(U, V)}\left(F_{I}^{\star}\right)\right)\left(F_{J}^{\star}-\mathbb{P}_{(U, V)}\left(F_{J}^{\star}\right)\right)\right)+\int_{D}\left\{v_{2}(s) \int_{(0,1) \times(0, s)} F_{I}^{\star}(u, v) \mathrm{d} C(u, v)\right\} \mathrm{d} s \\
& -\int_{D}\left\{v_{1}(s) \int_{(0, s) \times(0,1)} F_{I}^{\star}(u, v) \mathrm{d} C(u, v)\right\} \mathrm{d} s+\mathbb{P}_{(U, V)}\left(F_{I}^{\star}\right) \int_{D} s\left(v_{1}(s)-v_{2}(s)\right) \mathrm{d} s .
\end{aligned}
$$


Next straightforward computations yield

$$
\begin{aligned}
\sqrt{n}\left\{R_{n}(1,2)-R(1,2)\right\} & =\sqrt{n}\left\{\frac{\Delta J_{n}(1,2)}{\Delta I_{n}(1,2)}-\frac{\Delta J(1,2)}{\Delta I_{n}(1,2)}+\frac{\Delta J(1,2)}{\Delta I_{n}(1,2)}-\frac{\Delta J(1,2)}{\Delta I(1,2)}\right\} \\
& =\frac{1}{\Delta I_{n}(1,2)} \sqrt{n}\left\{\Delta J_{n}(1,2)-\Delta J(1,2)\right\}-\frac{\Delta J(1,2)}{\Delta I(1,2) \Delta I_{n}(1,2)} \sqrt{n}\left\{\Delta I_{n}(1,2)-\Delta I(1,2)\right\} \\
& =\frac{1}{\Delta I(1,2)}\left(\mathbb{G}\left(F_{J}^{\star}\right)+\int_{D}\left(v_{2}(s) \mathbb{G}\left(f_{2, s}\right)-v_{1}(s) \mathbb{G}\left(f_{1, s}\right)\right) \mathrm{d} s\right)-\frac{\Delta J(1,2)}{(\Delta I(1,2))^{2}} \mathbb{G}\left(F_{I}^{\star}\right)+o_{p}(1) .
\end{aligned}
$$

Then

$$
\sqrt{n}\left\{R_{n}(1,2)-R(1,2)\right\}=a\left(\mathbb{G}_{n}\left(F_{J}^{\star}\right)+\int_{D}\left(v_{2}(s) \mathbb{G}_{n}\left(f_{2, s}\right)-v_{1}(s) \mathbb{G}_{n}\left(f_{1, s}\right)\right) \mathrm{d} s\right)-b \mathbb{G}_{n}\left(F_{I}^{\star}\right)+o_{p}(1) .
$$

We finish by computing its variance $\Gamma(1,2)$. For this, let

$$
\begin{aligned}
& \mathbb{A}_{J}=\left(\mathbb{G}\left(F_{J}^{\star}\right)+\int_{D}\left(v_{2}(s) \mathbb{G}\left(f_{2, s}\right)-v_{1}(s) \mathbb{G}\left(f_{1, s}\right)\right) \mathrm{d} s\right), \\
& \mathbb{A}_{I}=\mathbb{G}\left(F_{I}^{\star}\right)
\end{aligned}
$$

and

$$
\Gamma(1,2)=\mathbb{E}\left(\left(a \mathbb{A}_{J}-b \mathbb{A}_{I}\right)^{2}\right)=a^{2} \mathbb{E}\left(\left(\mathbb{A}_{J}\right)^{2}\right)+b^{2} \mathbb{E}\left(\left(\mathbb{A}_{I}\right)^{2}\right)-2 a b \mathbb{E}\left(\mathbb{A}_{I} \mathbb{A}_{J}\right) .
$$

By using the notation of Theorem 4, where we introduced $a$ and $b$, we arrive at

$$
\Gamma(1,2)=a^{2} \Gamma_{J}(1,2)+b^{2} \Gamma_{I}(1,2)-2 a b \Gamma_{I, J}(1,2) .
$$

This completely achieves the proofs. 